Portland State University

PDXScholar

$12-2015$

\title{
Could Application of Column-Grid-Array (CGA) Technology Result in Inelastic-Strain-Free State-of- Stress in Solder Material?
}

\author{
Ephraim Suhir \\ Portland State University \\ Reza Ghaffarian \\ Jet Propulsion Laboratory \\ Johann Nicolics \\ Technische Universität Wien
}

Follow this and additional works at: https://pdxscholar.library.pdx.edu/mengin_fac

Part of the Mechanical Engineering Commons

Let us know how access to this document benefits you.

\section{Citation Details}

Suhir, E., Ghaffarian, R., and Nicolics, J. (2015). Could Application of Column-Grid-Array (CGA) Technology Result in Inelastic-Strain-Free State-of-Stress in Solder Material? Journal of Materials Science: Materials in Electronics. Volume 26, Issue 12, pp 10062-10067.

This Article is brought to you for free and open access. It has been accepted for inclusion in Mechanical and Materials Engineering Faculty Publications and Presentations by an authorized administrator of PDXScholar. Please contact us if we can make this document more accessible: pdxscholar@pdx.edu. 


\title{
Could application of column-grid-array (CGA) technology result in inelastic-strain-free state-of-stress in solder material?
}

\author{
Ephraim Suhir $^{1,2,4} \cdot$ Reza Ghaffarian $^{3} \cdot$ Johann Nicolics $^{4}$
}

Received: 22 June 2015/ Accepted: 25 August 2015/Published online: 7 September 2015

(C) Springer Science+Business Media New York 2015

\begin{abstract}
Physically meaningful and easy-to-use analytical stress model is developed for a short cylinder (beam) clamped at the ends and subjected to bending caused by the ends offset. The offset is due, in its turn, to an external lateral force that has to be determined from the known offset. It is envisioned that such a beam can adequately represent the state of stress in a column-grid-array (CGA) solder joint interconnection experiencing thermal loading due to the thermal expansion/contraction mismatch of the IC package and the printed circuit board (PCB). The CGA designs are characterized by considerably higher stand-off heights than ball-grid-array (BGA) systems. The offset $\Delta=l \Delta \alpha \Delta \mathrm{t}$ for a CGA solder joint located at the distance $l$ from the mid-cross-section of the package/PCB assembly (the neutral point (DNP)), can be determined, in an approximate analysis, as a product of this distance and the "external" thermal mismatch strain $\Delta \alpha \Delta$ t between the IC package and the printed circuit board (PCB). Here $\Delta \alpha$ is the difference in the effective coefficients of thermal expansion (CTE) of the PCB and package materials, and $\Delta \mathrm{t}$
\end{abstract}

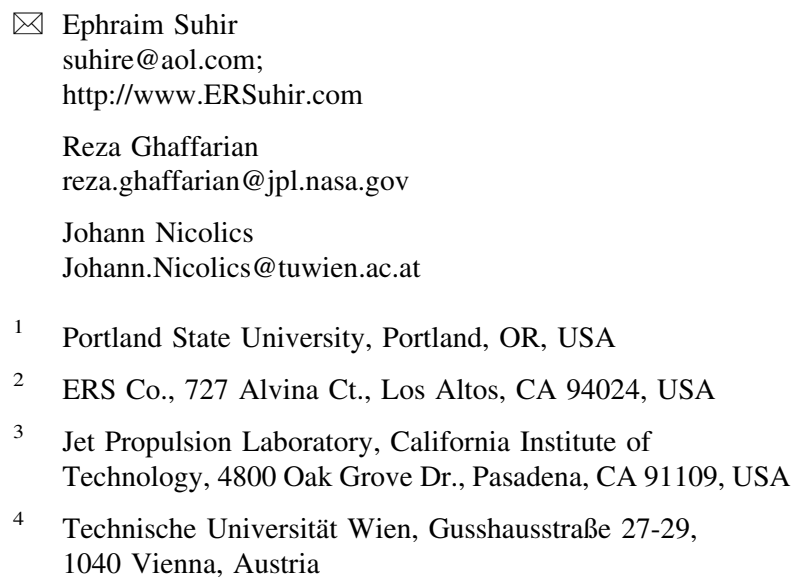

4 Technische Universität Wien, Gusshausstraße 27-29, 1040 Vienna, Austria

is the change in temperature. The objective of the analysis is to demonstrate that the application of a CGA design, in which the solder joints are configured as short clampedclamped beams, enables one not only to significantly relieve the thermally induced stresses, compared to the BGA system, but possibly to do that to an extent that the stresses in the solder material would remain within the elastic range. If this is achieved, the low-cycle-fatigue condition for the solder material will be replaced by the elastic-fatigue condition, thereby leading to a significantly longer fatigue lifetime of the joint. The elastic fatigue lifetime can be assessed, as is known, based on the Palmgren-Miner rule of linear accumulation of damages. Our analysis is limited therefore to elastic deformations.

\section{Introduction}

Ball-grid-array (BGA) is a widely used IC packaging technology [1-3]. It enables one to permanently surface mount electronic components onto a printed circuit board (PCB) with high mounting density (high pin count). In addition, BGA technology shortens signal delay. The long-term reliability of BGA systems is often insufficient, however, for many applications, and has been subject of numerous investigators [4-17]. Industry faces additional challenges with implementation of lead-free solder materials.

One important disadvantage of the BGA technology is that the BGA solder balls are not mechanically compliant. They do not flex the way the longer leads of the previous generations of the second level interconnections did and, because of that, are unable to effectively relieve stresses. Use of beam-like solder joints, such as column-grid-array (CGA) interconnections, for greater interfacial compliance is now implemented for high-reliability applications 
[18-22]. The problem was addressed independently [23] by using analytical modeling and considering the static behavior of the Timoshenko beam [24].

It should be emphasized that while the classical Timoshenko's short-beam theory seeks the beam's deflections caused by the combined bending and shear deformations for the given loading, an inverse problem is considered here: the lateral force and the corresponding stresses are sought for the given end offset. In short beams this force is larger than in long beams: in order to achieve the given displacement (offset), the applied force has to overcome both bending and shear resistance of the beam. Another difference is that the most indicative application of Timoshenko's theory is for a cantilever beam, while it is a clamped-clamped beam that mimics best solder joint geometry. The analysis that follows is, actually, a modification and an extension of the investigation [23], in which a short beam of a rectangular cross-section was addressed. Our objective is to show that the application of beam-likeconfigured solder joints could indeed lead to appreciably lower stresses in the material. In the current analysis a short cylinder is considered. We intend to find out if by application of beam-like solder joints one could not only just bring down the stress level, but to do that to an extent that no inelastic deformations could possibly occur.

It is noteworthy that avoiding inelastic-strains might be even possible with a conventional CBGA (ceramic BGA) technology [25], if a low expansion ceramic substrate, rather than a high-expansion plastic PCB, is employed [25]. In this case the thermal expansion (contraction) mismatch between the package and the substrate is relatively small, compared to a plastic PCB situation. It is noteworthy also that, as was shown earlier [26, 27] in application to Bell-Labs Si-on-Si flip-chip multi-chip packaging technology, solder joints configured as "pancakes" (i.e. those with large ratios of their diameter to the height) exhibited considerably higher stresses and strains than joints configured as "balls" (i.e. joints with lower aspect ratios). These observations are consistent with even more earlier findings [28-30], which indicated that by employing small size bonded assemblies with compliant interfaces or properly pre-engineered substrates with small "islands" at the interface with the vulnerable material one could bring down considerably the thermally or latticemismatch induced interfacial stresses. In these findings, however, the addressed assemblies and "islands" were characterized not by their height-to-width aspect ratios, but by the product $k l$ of the parameter $k$ of the interfacial shearing stress to half the assembly/ "island" length $l$. As to the height-to-width ratios of these assemblies/"islands", it was still expected that these ratios could be considerably less than one, i.e., that they were of a "pancake", rather than of a "ball" or a short-cylinder type addressed in this analysis.

\section{Analysis}

Consider a short cylinder (beam) of diameter $d$ and length (height) $h$. Beam's ends are clamped and offset at the given distance $\Delta$. The bending strain energy can be found as

$V_{b}=\frac{1}{2 E I} \int_{0}^{h} M^{2}(z) d z=\frac{32}{\pi E d^{4}} \int_{0}^{h} M^{2}(z) d z$,

where $E I$ is the beam's flexural rigidity, $E$ is Young's modulus of the material, $I=\frac{\pi d^{4}}{64}$ is the moment of inertia of the beam's cross-section,

$M(z)=M_{0}\left(1-\frac{2 z}{h}\right)$

is the distributed bending moment,

$M_{0}=\frac{1}{2} N_{0} h$

are the bending moments at the clamped ends, and $N_{0}$ is the lateral force. The origin of the vertical coordinate $z$ is at the beam's lower end.

The elastic curve $v(z)$ of the beam can be sought in the form of the method of initial parameters [30] as follows:

$v(z)=\frac{M_{0} z^{2}}{2 E I}-\frac{N_{0} z^{3}}{6 E I}=\frac{32 N_{0} z^{2}}{3 \pi E d^{4}}\left(\frac{3}{2} h-z\right)$.

The boundary condition $v(h)=\Delta$ yields:

$M_{0}=\frac{3 \pi E d^{4} \Delta}{32 h^{2}}, \quad N_{0}=\frac{3 \pi E d^{4} \Delta}{16 h^{3}}$

Then the Eq. (1) results in the following strain energy due to bending:

$V_{b}=\frac{3 \pi}{32} E \Delta^{2} \frac{d^{4}}{h^{3}}$

The strain energy due to shear (per unit beam's volume) can be sought as [30]:

$V_{s}^{\prime \prime}=\frac{3(1+v)}{2 E} \tau_{z x}^{2}$

Here $v$ is Poisson's ratio of the material, and $\tau_{z x}$ is the shearing stress associated with the distortion of the beam's shape. 
Let us assume that the shearing stress is distributed along the ultra-thin vertical strip of the elementary width $d r$ within beam's cross-section in a parabolic fashion

$\tau_{z x}=\tau_{\max }\left(1-\frac{x^{2}}{a^{2}}\right)$,

where $\tau_{\max }$ is the shearing stress at the center of the crosssection (i.e., the maximum shearing stress in the beam),

$a=a(r)=\sqrt{\frac{d^{2}}{4}-r^{2}}$

is half the strip's length, $0 \leq r \leq \frac{d}{2}$ is the distance of the strip from the center of the cross-section and $x$ is the distance of the given point of the strip from the horizontal diameter of the cross-section (where the strip's shearing stress is the maximum). The corresponding elementary lateral force $d N_{0}(r)$ can be found as

$$
\begin{aligned}
d N_{0}(r) & =d r \int_{-a}^{a} \tau_{z x}(x) d x d r=\tau_{\max } d r \int_{-a}^{a}\left(1-\frac{x^{2}}{a^{2}}\right) d x \\
& =\frac{4 a}{3} \tau_{\max } d r .
\end{aligned}
$$

The total lateral force is therefore

$N_{0}=\frac{4}{3} \tau_{\max } \int_{-d / 2}^{d / 2} \sqrt{\frac{d^{2}}{4}-r^{2}} d r=\frac{\pi}{6} d^{2} \tau_{\max }$.

The shearing strain energy of the strip can be found for the given radius $r$, based on the formulas (7) and (8), as follows:

$V_{s}^{\prime \prime}=\frac{3(1+v)}{2 E} \tau_{\max }^{2} \int_{-a}^{a}\left(1-\frac{x^{2}}{a^{2}}\right)^{2} d x=\frac{8}{5} \frac{1+v}{E} \tau_{\max }^{2} a(r)$.

The shearing strain for the entire cross-section is

$V_{s}^{\prime}=\frac{8(1+v)}{5 E} \tau_{\max }^{2} \int_{-d / 2}^{d / 2} \sqrt{\frac{d^{2}}{4}-r^{2}} d r=\frac{\pi}{5} \frac{1+v}{E} d^{2} \tau_{\max }^{2}$.

The shearing strain does not change along the beam and therefore for the entire beam this value should be simply multiplied by the beam's length (height) $h$ :

$V_{s}=\frac{\pi}{5} \frac{1+v}{E} d^{2} h \tau_{\max }^{2}$

Considering the formula (11) for the lateral force and the second formula in (5), the following formula for the strain energy due to shear can be obtained:
$V_{s}=\frac{36}{5 \pi} \frac{1+v}{E} \frac{h}{d^{2}} N_{0}^{2}=\frac{81 \pi}{320}(1+v) E \Delta^{2} \frac{d^{6}}{h^{5}}$.

Equating the total strain energy

$V=V_{b}+V_{s}=\frac{3 \pi}{32} E \Delta^{2} \frac{d^{4}}{h^{3}}\left[1+\frac{27}{10}(1+v)\left(\frac{d}{h}\right)^{2}\right]$

to the work $W=\frac{1}{2} N \Delta$ of the external lateral force $N$, the following formula for this force can be obtained:

$N=\frac{3 \pi}{16} E \Delta d\left(\frac{d}{h}\right)^{3}\left[1+\frac{27}{10}(1+v)\left(\frac{d}{h}\right)^{2}\right]$.

Comparing this formula with the second formula in (5), we conclude that the lateral force $N$ in the presence of shear deformations is larger by the factor of

$\chi=1+\frac{27}{10}(1+v)\left(\frac{d}{h}\right)^{2}$

than the force $N_{0}$ that does not consider these deformations. The factor (18) changes from 1.0 (for very thin-and-tall beams characterized by small diameter-to-height ratios) to about 1.90, when this ratio is as low as 0.5 (for Poisson's ratio of 0.33 ).

From the formulas (11) and (17) the following expressions for the maximum stresses can be obtained:

$$
\begin{aligned}
\tau_{\max } & =\frac{9}{8} E \frac{\Delta}{d}\left(\frac{d}{h}\right)^{3}\left[1+\frac{27}{10}(1+v)\left(\frac{d}{h}\right)^{2}\right], \\
\sigma_{\max } & =3 E \frac{\Delta}{d}\left(\frac{d}{h}\right)^{2}\left[1+\frac{27}{10}(1+v)\left(\frac{d}{h}\right)^{2}\right]=\frac{8}{3} \frac{h}{d} \tau_{\max } .
\end{aligned}
$$

As these formulas indicate, as long as a beam model is use, the (normal) bending stress always exceeds the shearing stress.

The calculated stress and energy ratios are shown in Table 1 versus length-to-diameter ratios. The table data confirm the well known circumstance that the role of shear does not have to be accounted for for length (height)-todiameter ratios above $12-15$, weather one seeks, as it is in the classical Timoshenko model, the displacement of a cantilever beam subjected to a force applied to the beam's end, or, like in this analysis, the maximum force and the corresponding stresses for the given ends off-set.

In the case of a rectangular cross-section the following formula for the lateral force was obtained [23] for a beam of unit width and with the dimension $2 l$ in the direction (plane) of bending: 
Table 1 Shear to tensile stress vs height to diamer

\begin{tabular}{lllllll}
\hline$h / d$ & 1 & 2 & 4 & 8 & 12 & 15 \\
\hline$\tau_{\max } / \sigma_{\max }$ & 0.37500 & 0.18750 & 0.09375 & 0.046875 & 0.03125 & 0.02500 \\
$V_{s} / V_{b}$ & 1.79550 & 0.44860 & 0.11222 & 0.02805 & 0.01247 & 0.00798 \\
\hline
\end{tabular}

$N=E \Delta\left(\frac{2 l}{h}\right)^{3}\left[1+\frac{9}{5}(1+v)\left(\frac{2 l}{h}\right)^{2}\right]$.

Comparing the formulas (17) and (20) we conclude that the effect of shear on the lateral force and the induced stresses is considerably, by a factor of 1.5 , greater in the case of a more realistic circular cross-section.

\section{Discussion}

Solders in BGA/CGA are unique since they provide both electrical interconnection and mechanical load-bearing element for attachment of package on PCB and often function as a critical heat conduit too. A solder joint in isolation is neither reliable nor unreliable; reliability has meaning only in the context of interconnections either within package or outside of package onto PCB. For BGA/CGA package assembly, it is critical to also determine the value of " $\Delta$ ".

As schematically shown in Fig. 1, three elements play key roles in defining reliability for CGA, global, local, and solder alloy. The characteristics of these three elementspackage (e.g., die, substrate, solder joint, underfill), PCB (e.g., polymer, $\mathrm{Cu}$, plated through hole, microvia), solder joints (e.g., via balls, columns) - together with the use conditions, the design life, and acceptance failure probability for the electronic assembly determine the reliability

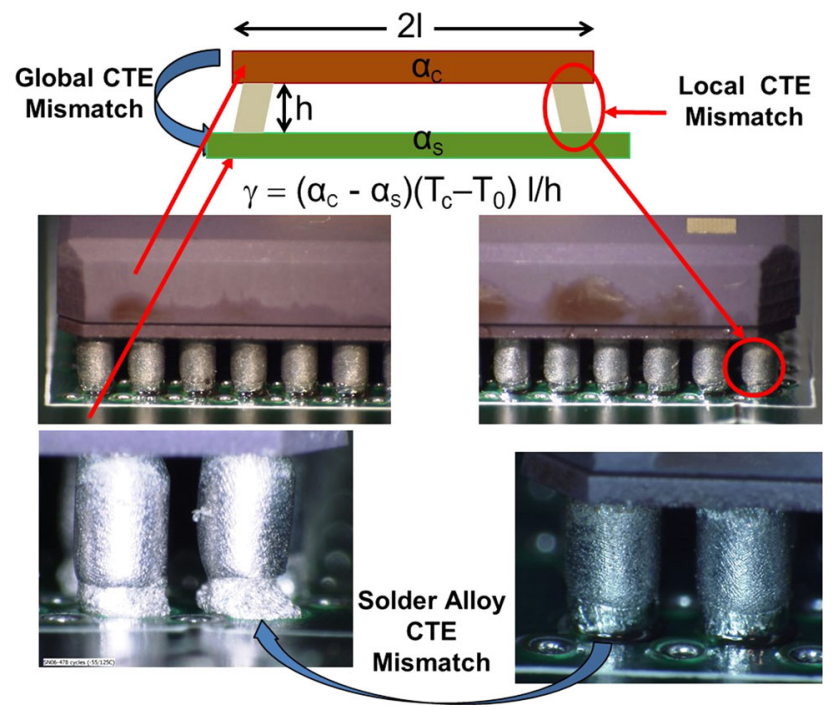

Fig. 1 Three key elements defines reliability under thermal stress are due to global, local, and solder alloy coefficient of thermal (CTE) mismatches of CGA assemblies. In addition, for CGA, solder columns also act as load carrying element between package and boards similar to metallic leads such as those for CQFP. Column flexibility and length to diameter ratio affect load bearing capability and are considered in the analyses.

The global expansion mismatches result from differential thermal expansions of a package and the PCB assembly. These thermal expansion differences $(\Delta)$ stem from differences in the coefficients of thermal expansion (CTEs) and thermal gradients as the result of heat dissipation from the functional die within the package. Global CTE-mismatches typically range from $\Delta \alpha \sim 2 \mathrm{ppm} /{ }^{\circ} \mathrm{C}\left(2 \times 10^{-6}\right)$ for CTEtailored high reliability assemblies to $\Delta \alpha \sim 14 \mathrm{ppm} /{ }^{\circ} \mathrm{C}$ for ceramic packages (e.g., CGA) on FR-4 PCBs. The thermal expansion mismatch representative of the global CTE mismatch due to thermal excursion is given as

$\Delta=\left(\alpha_{C}-\alpha_{S}\right)\left(t_{c}-t_{0}\right) l=(\Delta \alpha)(\Delta t) l$.

Global CTE mismatches typically are the largest, since all three parameters determining the thermal expansion mismatch, i.e., the CTE-mismatch $(\Delta \alpha)$, the temperature swing $(\Delta \mathrm{T})$, and the largest acting package diagonal length (2l), a.k.a., distance to neutral point (DNP), can be large.

The $\Delta$ plays a critical role in determination of shear tensile stresses in CGA columns; therefore, the analysis approach for a given end deformation were presented; whereas in the classical Timoshenko's short-beam theory seeks the beam's deflections caused by the combined bending and shear deformations for the given loading. The problem considered here determines the lateral force and the corresponding stresses for the given end offset. It is imperative, of course, that if CGA is employed for lower stresses, there is still enough interfacial real estate, so that the CGA bonding strength is not compromised. On the other hand, owing to the lower stress level, reliability assurance with CGA designs might be less of a challenge than in the case of BGA systems, even if the inelastic strains cannot be completely avoided, but occupies small peripheral portions of the assembly.

Let's calculate the shear and normal stresses for a typical high I/O CGA package with $35 \mathrm{~mm}^{2}$. The DNP for this package is from the center of package to the corner column is $\approx 25 \mathrm{~mm}$. Let's assume the CTE of the package and the $\mathrm{PCB}$ be $3 \times 10^{-6} /{ }^{\circ} \mathrm{C}$ and $18 \times 10^{-6} /{ }^{\circ} \mathrm{C}$, respectively, the change in temperature for a eutectic tin-lead solder from $183{ }^{\circ} \mathrm{C}$ to room temperature to $160{ }^{\circ} \mathrm{C}$. The induced 
Table 2 Shear, normal, and average stresses in CGA solder joints for a number of column height values

\begin{tabular}{lrrrl}
\hline$h(\mathrm{~mm})$ & 1 & 2 & 4 & 6.5 \\
\hline$\tau_{\max }\left(\mathrm{kg} / \mathrm{mm}^{2}\right)$ & 56.27 & 6.60 & 0.811 & 0.188 \\
$\sigma_{\max }\left(\mathrm{kg} / \mathrm{mm}^{2}\right)$ & 300.12 & 70.39 & 17.31 & 6.53 \\
$p\left(\mathrm{~kg} / \mathrm{mm}^{2}\right)$ & 315.55 & 71.32 & 17.37 & 6.54 \\
\hline
\end{tabular}

$\Delta=\left(18 \times 10^{-6}-3 \times 10^{-6}\right) \times 160 \times 25=0.060 \mathrm{~mm}$. Let the elastic constants of the solder material be $E=30 \mathrm{GPa}=3060 \mathrm{~kg} / \mathrm{mm}^{2}$ and $v=0.33$, the yield stress in tension $\sigma_{Y}=5.0 \mathrm{~kg} / \mathrm{mm}^{2}$, the diameter of the joint $\mathrm{d}=0.5 \mathrm{~mm}$. The computed maximum shearing and normal stresses $\tau_{\max }, \sigma_{\max }$, and the equivalent stress (the strain-energy based strength theory)

$p=\sqrt{\sigma_{\max }^{2}+3 \tau_{\max }^{2}}$

are shown in Table 2 for different heights (stand-offs) $h$ of the joint. The equivalent stress that corresponds to the state of stress at the yield point can be found, assuming $\tau_{Y}=$ $\frac{\sigma_{Y}}{\sqrt{3}}=2.887 \mathrm{~kg} / \mathrm{mm}^{2}, \quad$ as $\quad p_{Y}=\sqrt{\sigma_{Y}^{2}+3 \tau_{Y}^{2}}=\sqrt{2} \sigma_{Y}=$ $7.071 \mathrm{~kg} / \mathrm{mm}^{2}$. Considering Table 2 data, we conclude that the height (stand-off) of the joint of $h=6.5 \mathrm{~mm}$ will not lead to the occurrence of inelastic strains, i.e., to low cycle fatigue condition, and is therefore acceptable.

The carried out numerical example indicates that by employing beam-like CGA technology one could possibly manage to avoid inelastic strains in the solder material, and, hence, the low cycle fatigue conditions. If this is achievable, the fatigue lifetime of the solder material will be increased dramatically. This numerical example indicates also that the stand-off of a CGA joint should be rather large to keep the equivalent stress in an elastic condition.

It is noteworthy that the accuracy of some of the simplified assumption made in this analysis in order to obtain simple enough relationships need to be verified. A typical configuration of BGA and CGA package/assembly is shown in Fig. 2. This figure includes also representative of a cross-section for BGA and a scanning electron micrograph (SEM) of CGA after thermal cycling. As one could see, there are a few more or less obvious differences between the actual BGA and CGA configurations and that addressed in the analysis. Some other key elements to be considered in the future are:

- The $\Delta$ plays a critical role in determination of shear and tensile stresses in CGA columns; this in-turn depends on CTE, stiffness, and thickness of both package and PCB. These parameters need to be considered in the future analysis.

- The "clamp-clamp" condition is assumed in the analysis. As apparent from the CGA image, column could rotate during thermal cycling due to softer eutectic solder joint at the package/PCB interfaces. Futures analysis should consider this real condition, possibly as a composite material interaction.

- Future work should also include a specific test vehicle to capture details of analysis with verification with finite-element-analysis (FEA).

\section{Conclusions}

- The induced stresses in solder joints can be brought down considerably by employing CGA beam-like joints. It is imperative, of course, that if such joints are employed for lower stresses, there is still enough interfacial real estate so that the CGA bonding strength is not compromised. On the other hand, owing to a lower stress level in CGA systems with elevated standoff heights, assurance of their strength might be much less of a challenge than in the case of conventional BGA joints.

- By employing beam-like solder joints one might even avoid inelastic deformations in them, thereby increasing dramatically the lifetime of the material. The numerical example indicates particularly that the stand-
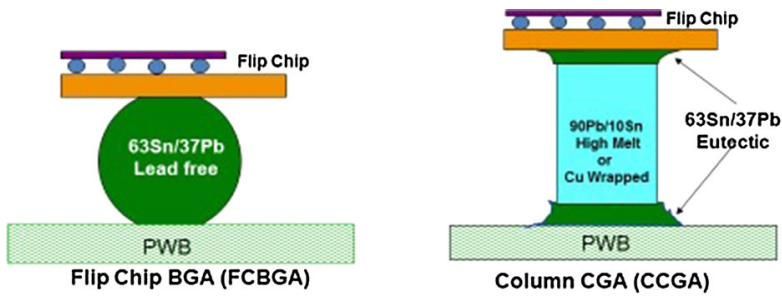

Column CGA (CCGA)
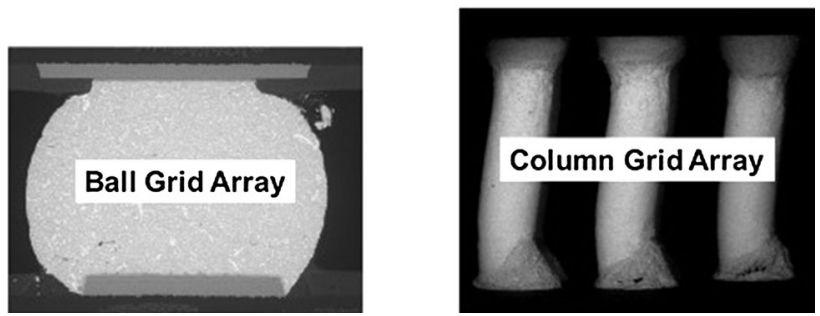

Fig. 2 Schematic ball grid array (BGA) and column grid array (CGA) and their representative images 
off of the CGA joint characterized by the inelasticstrain-free state-of-stress should be significant enough to make the shearing stress very low compared to the bending stress. This will take place for height-todiameter ratios of about 12-13. The further increase in the stand-off height, even if it is technologically achievable, is not recommended, since it will lead to undesirable elevated bending stresses.

- Future work will include, but might not be limited to, the finite-element-analysis (FEA) computations and experimental evaluations (such as, e.g., shear-off testing and/or temperature cycling) of the induced stresses in, and the fatigue lifetime of, a typical CGA joint.

Acknowledgments Part of the research described in this paper was conducted at the Jet Propulsion Laboratory, California Institute of Technology, under a contract with the National Aeronautics and Space Administration. The second author would like to acknowledge also the JPL and industry supports. The author also extends his appreciation to program managers of NASA Electronic Parts and Packaging (NEPP) Program.

\section{References}

1. J. Lau, Ball Grid Array Technology (McGraw-Hill, New York, 1994)

2. J. Lau, C. Wong, J. Prince, W. Nakayama, Electronic Packaging: Design, Materials, Process, and Reliability (McGraw-Hill, New York, 1998)

3. E. Suhir, C.P. Wong, Y.C. Lee (ed.) Micro- and Opto-Electronic Materials and Structures: Physics, Mechanics, Design, Packaging, Reliability, 2 volumes (Springer, 2008)

4. R. Ghaffarian, BGA assembly reliability, in Area Array Packaging Handbook, ed. by K. Gilleo (McGraw-Hill, New York, 2004)

5. R. Ghaffarian, Assembly and Reliability of $1704 \mathrm{I} / O$ FCBGA and FPBGAs, IPC APEX EXPO (2012)

6. R. Ghaffarian, Thermal cycle and vibration/drop reliability of area array package assemblies, in Structural Dynamics of Electronics and Photonic Systems, ed. by E. Suhir, E. Connally, D. Steinberg (Springer, New York, 2011)

7. J. Fjelstad, R. Ghaffarian, Y.G. Kim, Chip scale packaging for modern electronics, Electrochem. Publ. (2002)

8. Q. Yu, M. Shiratori, Y. Oshima, Thermal Fatigue Reliability Assessment for Solder Joints of BGA Assembly, The 11th Computational Mechanics Conference, JSME, No. 98-2, (1998), pp. $507-508$

9. J. Lau, Y. Pao, Solder Joint Reliability of BGA, CSP, Flip Chip, and Fine Pitch SMT Assemblies (McGraw-Hill, New York, 1997)

10. R. Darveaux, K. Banerji, A. Mawer, G. Dody, Reliability of Plastic Ball Grid Array Assemblies (McGraw-Hill, New York, 1995)

11. J. Lau, Z. Mei, S. Pang, C. Amsden, J. Rayner, S. Pan, Creep analysis and thermal-fatigue life prediction of the lead-free solder sealing ring of a photonic switch. ASME Trans. J. Electron. Packag. 124, 403-410 (2002)
12. Q. Zhang, A. Dasgupta, P. Haswell. Viscoplastic constitutive properties and energy-partitioning model of lead-free Sn3.9Ag0.6Cu solder alloy, IEEE ECTC, (2003)

13. J.Lau, W. Dauksher, P. Vianco, Acceleration models, constitutive equations and reliability of lead-free solders and joints, IEEE ECTC. 229-236 (2003)

14. A. Schubert, R. Dudek, E. Auerswald, A. Gollhardt, B. Michel, $\mathrm{H}$. Reicbl. Fatigue life models for $\mathrm{SnAgCu}$ and $\mathrm{SnPb}$ solder joints evaluated by experiments and simulation, IEEE ECTC, (2003)

15. A.R. Syed, Accumulated creep strain and energy density based thermal fatigue life prediction models for $\mathrm{SnAgCu}$ solder joints, IEEE ECTC, 737-746 (2004)

16. M. Osterman, A. Dasgupta, Life expectancies of Pb-Free SAC solder in-terconnects in electronic hardware. J. Master Sci. 18, 229-236 (2007)

17. B. Vandevelde, M. Gonzalez, P. Limaye, P. Ratchev, E. Beyne, Thermal cycling reliability of $\mathrm{SnAgCu}$ and $\mathrm{SnPb}$ solder joints: a comparison for several IC-packages. Microelectron. Reliab. 47, 259-265 (2007)

18. R. Ghaffarian, R., Damage and failures of CGA/BGA assemblies under thermal cycling and dynamic loadings, ASME 2013 International Mechanical Engineering Congress and Engineering. IMECE San Diego, California, USA, 15-21 Nov 2003

19. R. Ghaffarian, Thermal cycle reliability and failure mechanisms of CCGA and PBGA assemblies with and without corner staking, IEEE Trans. Compon. Packag. Technol. 31(2) (2008)

20. R. Ghaffarian, Area array technology for high reliability applications, in Micro-and Opto-Electronic Materials and Structures: Physics, Mechanics, Design, Reliability, Packaging, ed. by E. Suhir (Springer, New York, 2006)

21. R. Ghaffarian, CCGA packages for space applications. Microelectron. Reliab. 46, 2006-2024 (2006)

22. A.Tasooji, R. Ghaffarian, A, Rinaldi, Design Parameters Influencing Reliability of CCGA Assembly: Sensitivity Analysis, IEEE ITHERM Conference (2006)

23. E.Suhir, Analysis of a short beam with application to solder joints: could larger stand-off heights relieve stress?, Eur. Phys. J. Appl. Phys. (EPJAP), (2015), in print

24. S.P. Timoshenko, On the correction factor for shear of the differential equation for transverse vibrations of bars of uniform cross-section, Philos. Mag. 744 (1921)

25. E. Suhir, L.Bechou, B. Levrier, D. Calvez, Assessment of the Size of the Inelastic Zone in a BGA Assembly, IEEE Aerospace Conference (Big Sky, Montana, 2013)

26. E. Suhir, Axisymmetric elastic deformations of a finite circular cylinder with application to low temperature strains and stresses in solder joints, ASME J. Appl. Mech. 56(2) (1989)

27. E. Suhir, Mechanical Reliability of Flip-Chip Interconnections in Silicon-on-Silicon Multichip Modules, IEEE Conference on Multichip Modules, IEEE (Santa Cruz, California 1993)

28. E. Suhir, Stresses in Bi-Metal Thermostats, ASME J. Appl. Mech., 53(3) (1986)

29. S. Luryi and E. Suhir, "A new approach to the high-quality epitaxial growth of lattice-mismatched materials, Appl. Phys. Lett. 49(3) (1986)

30. E. Suhir, Structural Analysis in Microelectronic and Fiber Optic Systems, Basic principles of Engineering Elasticity and Fundamentals of Structural Analysis (van Nostrand Reinhold, New York, 1991) 